\title{
A SHORT NOTE ON HARMONIC FUNCTIONS ON SELF-SIMILAR STRUCTURES
}

\author{
BRIGITTE E. BRECKNER
}

\begin{abstract}
A sufficient condition is given concerning the harmonic structure on a post critically finite self-similar structure $K$ that ensures that harmonic functions are not zero divisors in the algebra of real-valued continuous functions on $K$.

MSC 2010. 28A80, 49J52.
\end{abstract}

Key words. Self-similar structure, harmonic function, Sierpinski fractal.

\section{REFERENCES}

[1] B.E. Breckner, Real-valued functions of finite energy on the Sierpinski gasket, Mathematica, (55) 78 (2013), 2, 142-158.

[2] B.E. Breckner, A short note on harmonic functions and zero divisors on the Sierpinski fractal, Arch. Math. (Basel), 106 (2016), 183-188.

[3] B.E. Breckner and R. Chill, The Laplace operator on the Sierpinski gasket with Robin boundary conditions, Nonlinear Anal. Real World Appl., 38 (2017), 245-260.

[4] B.E. Breckner and Cs. Varga, Multiple solutions of Dirichlet problems on the Sierpinski gasket, J. Optim. Theory Appl., 167 (2015), 3, 842-861.

[5] B.E. Breckner and Cs. Varga, An application of a fixed-point theorem to Neumann problems on the Sierpinski fractal, Fixed Point Theory, 19 (2018), 2, 475-486.

[6] M. Galewski, Multiple Solutions to a Dirichlet problem on the Sierpinski gasket, Taiwanese J. Math., 20 (2016), 5, 1079-1092.

[7] M. Galewski, Optimization problems on the Sierpinski gasket, Electron. J. Differential Equations, 2016, 105, 1-11.

[8] J. Kigami, A harmonic calculus on the Sierpinski spaces, Japan J. Appl. Math., 6 (1989), 259-290.

[9] J. Kigami, Harmonic calculus on p.c.f. self-similar sets, Trans. Am. Math. Soc., 335 (1993), 721-755.

[10] J. Kigami, Analysis on Fractals, Cambridge University Press, Cambridge, UK, 2001.

[11] G. Molica Bisci and V. Rădulescu, A characterization for elliptic problems on fractal sets, Proc. Amer. Math. Soc., 143 (2015), 7, 2959-2968.

[12] G. Molica Bisci, D. Repovš and R. Servadei, Nonlinear problems on the Sierpinski gasket, J. Math. Anal. Appl., 452 (2017), 2, 883-895.

[13] R.S. Strichartz, Differential Equations on Fractals. A Tutorial, Princeton University Press, Princeton, NJ, 2006.

Received December 3, 2019

Accepted March 25, 2020

DOI: 10.24193/mathcluj.2020.2.05 
Babeş-Bolyai University

Faculty of Mathematics and Computer Science

Str. M. Kogălniceanu nr. 1

400084 Cluj-Napoca, Romania

E-mail: brigitte@math.ubbcluj.ro 